\title{
Conservative surgical management of in situ subungual melanoma: long-term follow-up*
}

\author{
Mariana Catalina De Anda-Juárez ${ }^{1}$ \\ Verónica Fonte-Ávalos ${ }^{1}$ \\ Judith Domínguez-Cherit²
}

\author{
María Abril Martínez-Velasco ${ }^{1}$ \\ Sonia Toussaint-Caire ${ }^{1}$
}

DOI: http://dx.doi.org/10.1590/abd1806-4841.201645100

\begin{abstract}
Subungual melanoma represents $20 \%$ of all melanomas in Hispanic population. Here, we report the outcome of 15 patients with in situ subungual melanoma treated with resection of the nail unit with a 5-mm margin without amputation, followed up for $55.93 \pm 43.08$ months. The most common complications included inclusion cysts and nail spicules. We found no evidence of local or distant recurrences at the last visit of our follow up. Functional outcome was good, with only one patient reporting persistent mild pain. These results support functional, non-amputative surgical management of in situ subungual melanomas.
\end{abstract}

Keywords: Follow-up studies; Melanoma; Nail diseases

Subungual melanoma (SUM) is a subtype of acral melanoma. It represents $20 \%$ of all melanomas in Hispanic population and is the fourth most common nail tumor in our department. ${ }^{1}$ Prognosis and metastasis rates of SUM mainly depend on Breslow thickness as in other variants of melanoma. ${ }^{2}$ However, SUM is more often diagnosed in advanced stages. ${ }^{3}$

In early stages (radial growth), SUM presents as an irregular band of longitudinal melanonychia, sometimes with Hutchinson's sign (pigment extension around the nail apparatus). This clinical presentation generally corresponds to in situ or minimally invasive melanoma (less than $0.5 \mathrm{~mm}$ in Breslow depth). ${ }^{3}$ In more advanced stages, it is usually accompanied by nail dystrophy or can even become amelanotic, making the diagnosis more difficult. ${ }^{4}$ Dermoscopy reveals irregular deposition of pigment globules, irregular bands of melanonychia, and micro-Hutchinson sign. ${ }^{5}$ The gold standard for the diagnosis of SUM is nail matrix biopsy either by punch, tangential shaving, incisional nail matrix biopsy or lateral longitudinal biopsy. ${ }^{6}$ Recommended treatment consists of surgical excision and depends mainly on Breslow thickness.

The aim of the present study was to describe the clinical presentation, evolution, surgical technique, and outcome of a cohort of patients with in situ SUM treated with removal of the nail unit with $5 \mathrm{~mm}$ margins.
This study was conducted in the Dermatology Department of Hospital General Dr. Manuel Gea González in Mexico City. We performed a search in our histopathology database in order to retrieve all patients with diagnosis of in situ SUM from 1989 to 2013. All cases were confirmed by biopsy and treated with surgical excision of the nail unit with a $5 \mathrm{~mm}$ margin. Data obtained from records included: demographic data; time of evolution before final diagnostic biopsy; previous diagnosis and treatments; history of trauma; topography; clinical presentation; presence of Hutchinson's sign; nail dystrophy; dermoscopy (when available); and type of reconstruction.

Fifteen patients ( 9 females and 6 males; age $31.67 \pm 20.9$, range 4-66 years) were included and assessed with a physical exam and clinical photographs. Time of evolution until diagnostic biopsy was $79.4 \pm 48.25$ months (3 previously diagnosed as having naevi, 2 as racial melanonychia, and 1 treated as onychomycosis).

The most common clinical presentation was longitudinal melanonychia measuring 2-6 mm wide in 11 patients; with irregular distribution of pigment globules on dermoscopy. The 4 remaining presented with total melanonychia. Hutchinson's sign was present in 7 cases, and 5 patients had nail plate dystrophy, consisting of pachionychia and longitudinal riding. None of the patients reported history of trauma.

Received on 02.09.2015

Approved by the Advisory Board and accepted for publication on 28.10.2015

* Work performed at the Department of Dermatologic Surgery, Hospital General Dr. Manuel Gea González. Mexico City, Mexico.

Financial Support: None.

Conflict of Interest: None. 

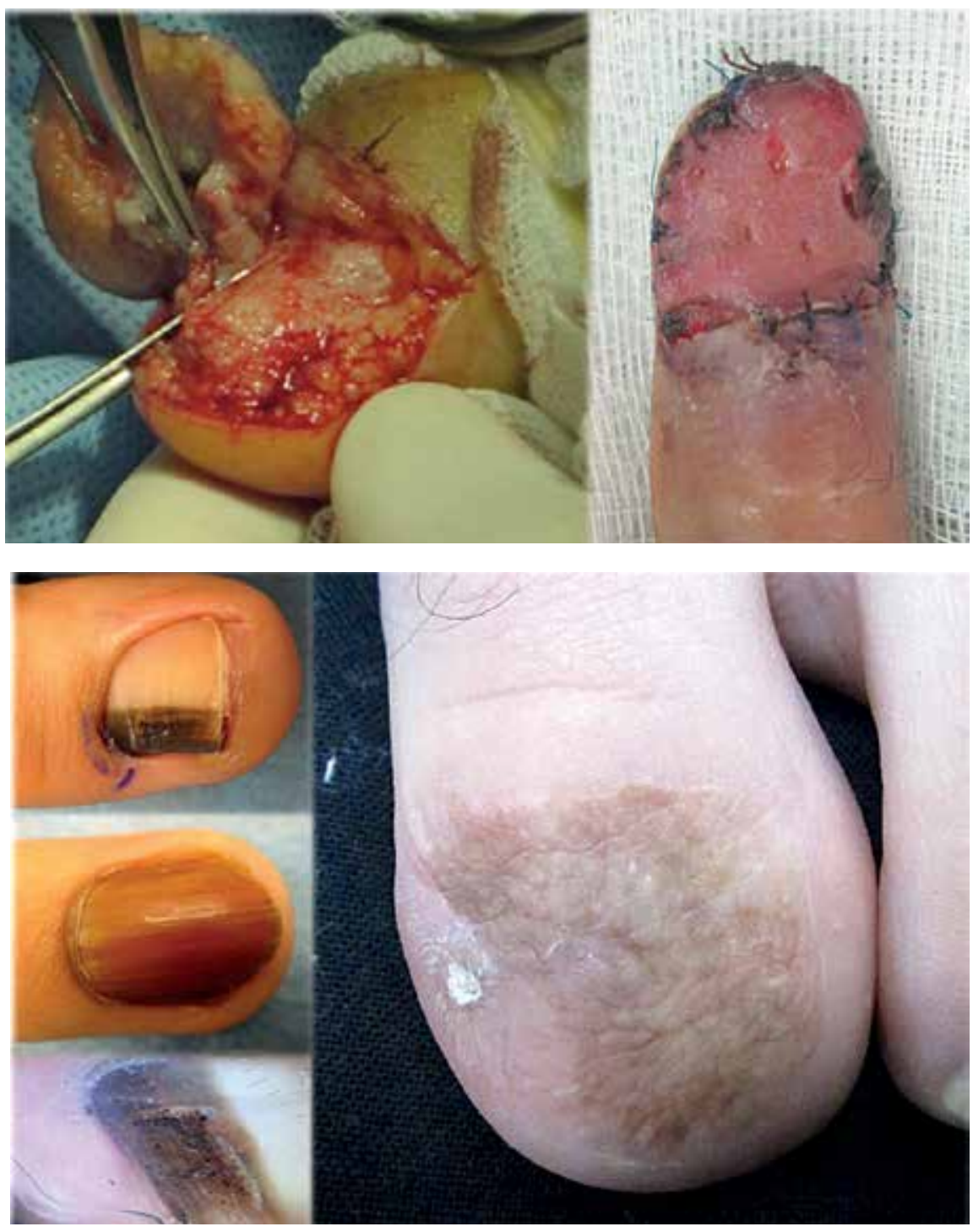

FigURE 1:

5-mm margin resection of the nail unit and reconstruction with full thickness skin graft

\section{FIGURE 2:}

In-situ SUM: longitudinal melanonychia and total melanonychia. Dermoscopy shows irregular distribution of pigment globules. Hyperpigmentation of the graft and nail spicule on the lateral border
The right hand was involved in 5 patients ( 2 patients in the first finger, 2 patients in the fourth finger and one in the fifth finger), and the left hand in 4 patients ( 1 patient in the first finger and 3 in the fifth finger). The lower extremities were involved in the remaining 6 patients; 5 cases in the first finger of the right foot and one in the first finger of the left foot.

All patients were treated with complete removal of the nail apparatus with a $5 \mathrm{~mm}$-margin and supra-periosteum depth resection. Fourteen patients were reconstructed with a full thickness skin graft, and one patient was treated with a banner flap (Figure 1).

Follow up after surgical treatment was $55.93 \pm 43.08$ months (range 15 to 159 months). The most common complications were: nail spicules in 6 cases, inclusion cysts in 4 cases, persistent hypersensitivity of the digit in 4 patients, and persistent moderate pain when walking referred by one patient. Functional and cosmetic outcome was good in all of them with hyperpigmentation of the skin graft in 9 patients (Figure 2).

At the moment of this report, none of this patients presented local or distant tumor recurrence.
In the present series of patients, conservative management of in situ SUM with wide resection of the nail unit without amputation resulted in good functional and cosmetic outcomes in the long term, without any local or distant tumor recurrence. Complications of the surgical procedure were minor and only one patient had persistent pain. These results support wide resection treatment without amputation in patients with in situ SUM.

Recent studies by Lazar and Sureda support conservative or functional excision without amputation with wide margin resection of the nail unit in SUM and no recurrence after 2-4 years follow-up. ${ }^{4,7}$

Our findings support functional surgical treatment of patients with in situ SUM with a safe and good, result with only mild complications, without any recurrence after a minimal 15-month follow-up. This is a small series in Hispanic population that contributes by reporting results without amputation. Larger series and longer follow-up periods would contribute to confirm the safety of this procedure. $\square$ 


\section{REFERENCES}

1. Domínguez-Cherit J, Chanussot-Deprez C, Maria-Sarti H, Fonte-Avalos V, VegaMemije E, Luis-Montoya P. Nail unit tumors: a study of 234 patients in the dermatology department of the "Dr Manuel Gea Gonzalez" General Hospital in Mexico City. Dermatol Surg. 2008;34:1363-71.

2. Phan A, Touzet S, Dalle S, Ronger-Savlé S, Balme B, Thomas L. Acral lentiginous melanoma: a clinicoprognostic study of 126 cases. Br J Dermatol. 2006;155:5619.

3. Rex J, Paradelo C, Mangas C, Hilari JM, Fernández-Figueras MT, Ferrándiz C. Management of primary cutaneous melanoma of the hands and feet: a clinicoprognostic study. Dermatol Surg. 2009;35:1505-13.

4. Sureda N, Phan A, Poulalhon N, Balme B, Dalle S, Thomas L. Conservative surgical management of subungual (matrix derived) melanoma: report of seven cases and literature review. Br J Dermatol. 2011;165:852-8.

5. Phan A, Dalle S, Touzet S, Ronger-Savlé S, Balme B, Thomas L. Dermoscopic features of acral lentiginous melanoma in a large series of 110 cases in a white population. Br J Dermatol. 2010;162:765-71.

6. Braun RP, Baran R, Le Gal FA, Dalle S, Ronger S, Pandolfi R, et al. Diagnosis and management of nail pigmentations. J Am Acad Dermatol. 2007;56:835-47.

7. Lazar A, Abimelec $P$, Dumontier C. Full thickness skin graft for nail unit reconstruction. J Hand Surg Br. 2005;30:194-8.
MAILING ADDRESS:

Mariana Catalina De Anda Juárez

Department of Dermatologic Surgery,

Hospital General Dr. Manuel Gea González

Calzada de Tlalpan 4800, Colonia XVI, Tlalpan,

14080 Mexico City, Mexico

E-mail:mdeanda73@gmail.com

How to cite this article: De Anda-Juárez M, Martínez-Velasco MA, Fonte-Ávalos V, Toussaint-Caire S, DomínguezCherit J. Conservative surgical management of in situ subungual melanoma: long-term follow-up. An Bras Dermatol. 2016;91(6):846-8. 